\title{
A VISUALIDADE NA EDUCAÇÃO DE SURDOS: UMA REVISÃO SISTEMÁTICA DA LITERATURA
}

\author{
VISUALITY IN DEAF EDUCATION: A SYSTEMATIC REVIEW
}

Welbert Vinícius de Souza Sansão ${ }^{1}$, Anabela Cruz-Santos ${ }^{2}$

Submetido em: $21 / 04 / 2020$

Avaliado em: 05/06/2020

Aprovado em: 23/02/2020

\section{RESUMO}

Este artigo objetiva realizar um levantamento de pesquisas sobre Educação de Surdos atendendo-se à visualidade como elemento de mediação no processo de aprendizagem. Foi realizada uma pesquisa bibliográfica nas bases de dados de indexação de artigos, no qual selecionamos as plataformas da Scientific Eletronic Library Online (Scielo) e o Portal de Periódicos da CAPES. Como procedimentos metodológicos, subdividimos em cinco etapas: (1) definição da estratégia de busca, na qual optamos pelos termos "visualidade", "aprendizagem" e "surdos" em três campos de busca (título, resumo, palavras-chaves); (2) busca por artigos científicos nas duas bases de pesquisa; (3) seleção dos estudos com aplicação de critérios de seleção e exclusão; (4) análise da coleta e extração de dados e (5) considerações quanto às oportunidades de pesquisa. Foram encontradas, inicialmente, noventa e cinco artigos que tratavam sobre visualidade, em seguida foi realizada uma nova análise considerando, neste momento, apenas as que referenciavam em suas pesquisas os processos de aprendizagem de estudantes Surdos, assim, foram detectados cinco artigos. Com a análise dos resultados, verificamos que os artigos selecionados apontavam a visualidade como elemento mediador da aprendizagem de Surdos, visto que os Surdos apreendem o mundo e interagem com ele a partir das experiências visuais. Assim, concluímos que se faz necessário explorar o uso e a aplicabilidade dos recursos visuais na Educação de Surdos, a fim de explorar as potencialidades instrumentais desses sujeitos para o desenvolvimento do pensamento.

PALAVRAS-CHAVE: Educação de Surdos. Visualidade. Aprendizagem.

${ }^{1}$ Doutorando em Estudos da Criança pela Universidade do Minho. Mestre em Educação pela Universidade Federal de Lavras com enfoque na Educação Matemática para Surdos. Especialista em Libras e graduado em Licenciatura em Matemática. Proficiência na Tradução e Interpretação de Libras (2010), bem como, Proficiência em Ensino da Libras (2015). Formação como Intérprete e Tradutor da Língua Brasileira de Sinais pelo CAS/MEC/BH. Atualmente, é Professor de Libras na Universidade Federal Recôncavo da Bahia (UFRB). Desenvolve pesquisas na área de Educação de Surdos, Educação Matemática e Estudos da Tradução. Membro do Grupo de Pesquisa em Práticas Pedagógicas orientadas pela Teoria Histórico-Cultural e do Núcleo de Estudos e Pesquisas em Interiorização da Língua de Sinais (Neplis). Correio eletrônico: welbert.sansao@gmail.com

²Departamento de Psicologia da Educação e Educação Especial , Instituto de Educação, Universidade do Minho.

Doutorada em Estudos da Criança, especialização em Educação Especial pela Universidade do Minho. Investigadora integrada do Centro de Investigação em Educação (CIEd). Leciona UC relacionadas com as Necessidades Educativas Especiais, Problemas de Comunicação, e Avaliação desde as idades precoces até à idade escolar. Leciona desde 2000 em cursos de formação de professores de Educação Especial e outros profissionais. Tem coordenado e participado em projetos nacionais e internacionais na área das Perturbações da Linguagem. Principais interesses de investigação: aquisição e desenvolvimento de competências de comunicação em crianças com e sem Necessidades Educativas Especiais, construção e validação de instrumentos de avaliação de linguagem, e estratégias baseadas na investigação para crianças com Perturbações da Comunicação, Deficiência Auditiva e Deficiência Visual. Correio eletrônico: acs@ie.uminho.pt 


\begin{abstract}
This article aims to conduct a survey of research on Education for the Deaf given the visuality as an element of mediation in the learning process. A bibliographic search was carried out in the article indexing databases, in which we selected the platforms of the Scientific Eletronic Library Online (Scielo) and the CAPES Journal Portal. As methodological procedures, we subdivided into five stages: (1) definition of the search strategy, in which we chose the terms "visuality", "learning" and "deaf" in three search fields (title, abstract, keywords); (2) search for scientific articles in the two research bases; (3) selection of studies with application of selection and exclusion criteria; (4) analysis of data collection and extraction and (5) considerations regarding research opportunities. Initially, ninety-five articles dealing with visuality were found, then a new analysis was carried out considering, at this moment, only those that referenced the learning processes of Deaf students in their research, thus, five articles were detected. With the analysis of the results, we found that the selected articles pointed to visuality as a mediating element in the learning of the Deaf, since the Deaf apprehend the world and interact with it from visual experiences. Thus, we conclude that it is necessary to explore the use and applicability of visual resources in the Education of the Deaf, in order to explore the instrumental potential of these subjects for the development of thought.
\end{abstract}

KEYWORDS: Deaf Education. Visuality. Learning. 


\section{INTRODUÇÃO}

O momento sócio-histórico atual no qual ações governamentais decorrentes ao movimento da comunidade, têm sido implementadas. Diante de um embate de forças, em 2002, foi sancionada a Lei 10.436, posteriormente regulamentada pelo Decreto 5.626/05 (Brasil, 2002, 2005). Portanto, vale ressaltar que uma importante questão relacionada ao campo de Educação de Surdos refere-se ao uso e difusão da Libras, como língua de instrução. Esses textos jurídicos trazem importantes avanços concernentes aos direitos dos surdos, dentre eles, o direito à educação bilíngue. Nesse sentido, os incisos abaixo tratam dessa matéria:

Art. 22. As instituições federais de ensino responsáveis pela educação básica devem garantir a inclusão de alunos surdos ou com deficiência auditiva, por meio da organização de: I - escolas e classes de educação bilíngue, abertas a alunos surdos e ouvintes, com professores bilíngues, na educação infantil e nos anos iniciais do ensino fundamental;

II - escolas bilíngues ou escolas comuns da rede regular de ensino, abertas a alunos surdos e ouvintes, para os anos finais do ensino fundamental, ensino médio ou educação profissional, com docentes das diferentes áreas do conhecimento, cientes da singularidade linguística dos alunos surdos, bem como com a presença de tradutores e intérpretes de Libras - Língua Portuguesa (Brasil, 2005).

O atual Plano Nacional de Educação (PNE), Lei $13.005 / 14$, que, em sua elaboração contou com uma intensa participação da comunidade surda, contemplou em uma de suas estratégias (Meta 4.7) a reafirmação do direito do estudante surdo da rede regular de ensino frequentar escolas bilíngues ${ }^{3}$ e às escolas inclusivas (Brasil, 2014).

Portanto, percebemos que o estudante surdo no Brasil está bem amparado diante dos dispositivos legais, no entanto, maior visibilidade não qualifica práticas pedagógicas ideais no processo de aprendizagem, isto porque, para Borges (2013), ainda faltam atividades e instrumentos que explorem a visualidade do Surdo, principalmente na Educação.

Como afirma Reily (2003), a visualidade, explorada como instrumento, traz consigo o potencial de mediação que tenciona a formação do pensamento. Para o estudante Surdo, esse caminho para o desenvolvimento será necessariamente visual, isto porque o universo de sua língua e a maneira que interagem como o mundo é puramente visual.

Os estudos de Vygotsky (1998) perpassam por três fundamentos do pensamento: condição biológica, a consciência advinda das práticas sociais aliada ao processo histórico e a relação sujeito mediados por instrumento e signos. A partir dessa afirmativa que postula que as funções psíquicas superiores surgem da articulação entre os instrumentos e os signos. Nesse contexto, percebe-se a importância de se repensar e aprimorar métodos para a Educação de Surdos a fim de explorar os artefatos visuais (instrumentos) e língua de sinais (signos), como elementos mediativos tencionadores da aprendizagem.

Como a língua é um produto de significações que organiza as funções psíquicas superiores e permite que o ser humano desenvolva seu pensamento, a Libras, conforme explicitado por Campello (2008), é tida como signo visual que estudantes Surdos a utilizam no processo de significação, possibilitando, por sua vez, a abstração, generalização e a categorização/conceitual. Explorar as habilidades e percepções visuais pode propiciar uma ligação entre significado e significante, entre conteúdo e prática, portanto, a prática pedagógica persiste em criar estratégias metodológicas que estimulem os Surdos a fazer mapas mentais para que o aprendizado consolide, partindo do pressuposto que a língua natural dos Surdos é na modalidade viso-espacial (Marques, 1999). Neste sentido, as especificidades dos Surdos precisam ser levadas em conta, ao se pensar em Educação de Surdos.

Nessa perspectiva, com o objetivo de investigar os estudos relacionados à visualidade e as potencialidades no processo de aprendizagem, foi realizada uma

\footnotetext{
${ }^{3} \mathrm{O}$ bilinguismo é uma proposta de educação usada por escolas que se propõem a tornar acessível à criança surda duas línguas no contexto escolar. Os estudos têm apontado essa proposta como sendo a mais adequada para o ensino de crianças surdas, tendo em vista que considera a língua de sinais como primeira língua e parte desse pressuposto para o ensino da língua escrita. (Quadros, 1997)
} 
revisão sistemática da literatura (RSL) sobre o que tem sido produzido nas pesquisas que perpassam essa temática.

\section{PROCEDIMENTOS METODOLÓGICOS}

Com o intuito de compreender o que as pesquisas abordam quanto aos aspectos de visualidade no processo de aprendizagem de estudantes Surdos, realizou-se uma RSL para auxiliar na busca e reflexão por conhecimentos direcionados e atualizados nesta área. Segundo Felizardo et al (2017), a RSL é um método sistemático que objetiva buscar, selecionar e avaliar criticamente os estudos.

Assim, como procedimentos metodológicos, subdividimos em cinco etapas: (1) definição da estratégia de busca; (2) busca por artigos científicos em duas bases de pesquisa; (3) seleção dos estudos com aplicação de critérios de seleção e exclusão; (4) análise da coleta e extração de dados e (5) considerações quanto às oportunidades de pesquisa. Adotamos esse método, pautando-se dos estudos de Felizardo et al (2017), que a caracteriza conforme figura abaixo.

Figura 1 - Etapas metodológicas no processo de coleta e análise.

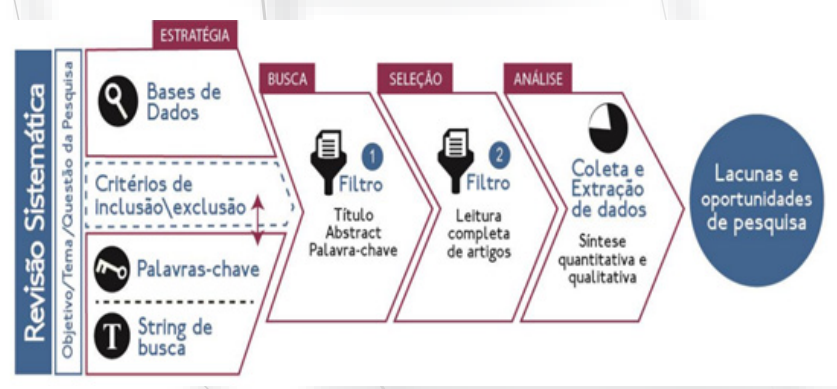

Fonte: Felizardo et al (2017).

Em primeiro momento, norteados pela seguinte pergunta de pesquisa: $\mathrm{O}$ que as pesquisas abordam quanto as potencialidade da visualidade no processo de aprendizagem de estudantes Surdos?. Estrategicamente selecionamos a plataforma Scientific Eletronic Library Online (Scielo) e o "Catálogo de Periódicos da CAPES", na qual realizamos um levantamento de dados, a partir da definição do tema, do objetivo e da questão de pesquisa formulada. Permitindo, portanto, a parametrização das buscas, a fim de montar a string completa (quadro
1) e iniciar a catalogação dos artigos.

Quadro 1 - Escolha dos Termos

\begin{tabular}{|c|l|}
\hline \multicolumn{2}{|c|}{ Seleção de Termos estratégicos } \\
\hline 1 & Visualidade; \\
\hline 2 & Surdos; \\
\hline 3 & Aprendizagem. \\
\hline
\end{tabular}

Fonte: O Autor

A partir da escolha dos três termos, realizamos o filtro em 3 campos de busca (título, resumo, palavras-chaves), no período de 13 de janeiro a 19 de janeiro de 2020. Foram encontrados noventa e cinco artigos considerando o critério inicial de seleção e a correlação com os termos definidos.

Posteriormente, localizamos o texto na íntegra de todas as pesquisas e organizamos os dados em uma tabela com autor, título, objetivos da pesquisa, referenciais teóricos utilizados, metodologia adotada e resultados. Para adquirir esses dados consultamos os resumos e, quando necessário, a leitura na íntegra dos artigos. A seguir, apresentamos brevemente cada estudo e, em seguida, tecemos algumas considerações sobre eles.

\section{ANÁLISE DOS RESULTADOS}

Ao realizarmos o levantamento de artigos que perpassam a temática deste escopo, encontramos onze pesquisas, dentre essas selecionamos apenas aquelas que abordavam quanto as correlações do processo de aprendizagem e as potencialidades da visualidade, dessas apenas quatro tratavam desta temática. No quadro 2 a seguir, são apresentados os autores, títulos, objetivos e os anos de publicação dos estudos. 
Quadro 2 - Artigos Selecionados na Pesquisa nas Bases de Dados

Fonte: O Autor

\begin{tabular}{|c|c|c|c|}
\hline \multicolumn{4}{|c|}{ Artigos } \\
\hline Autores & Titulo & Objetivo & Ano \\
\hline $\begin{array}{l}\text { Pivetta, E. M. } \\
\text { Saito, D. S. } \\
\text { Almeida, A. M. P. } \\
\text { Ulbricht, V. R. }\end{array}$ & $\begin{array}{c}\text { Contribuições para o } \\
\text { design de interface de um } \\
\text { Ambiente Virtual de Ensino } \\
\text { Aprendizagem acessível a } \\
\text { surdos }\end{array}$ & $\begin{array}{l}\text { Apresentar os resultados de } \\
\text { uma investigação com } \\
\text { profissionais da educação } \\
\text { de uma escola bilíngue para } \\
\text { surdos em Portugal }\end{array}$ & 2013 \\
\hline Sales, E. R. & $\begin{array}{l}\text { A visualização no ensino } \\
\text { de matemática: uma } \\
\text { experiência com alunos } \\
\text { surdos }\end{array}$ & $\begin{array}{l}\text { Investigar de que forma a } \\
\text { visualidade da pessoa surda } \\
\text { pode contribuir para o ensino e } \\
\text { aprendizagem de matemática. }\end{array}$ & 2017 \\
\hline $\begin{array}{c}\text { Freitas-Reis, I. } \\
\text { Fernandes, J. M. } \\
\text { Carvalho, V. } \\
\text { Franco-Patrocínio, S. } \\
\text { Faria, F. L. }\end{array}$ & $\begin{array}{l}\text { Métodos de avaliação para } \\
\text { o aluno surdo no contexto } \\
\text { do ensino de química }\end{array}$ & $\begin{array}{c}\text { Propor atividades de avaliação } \\
\text { escolar para alunos surdos no } \\
\text { contexto do ensino de } \\
\text { química. }\end{array}$ & 2017 \\
\hline $\begin{array}{l}\text { Paiva, R. O. } \\
\text { Benchimol, A. } \\
\text { Chalhub, T. }\end{array}$ & $\begin{array}{c}\text { Breves apontamentos } \\
\text { sobre um repositório digital } \\
\text { bilíngue (Português-Libras): } \\
\text { o caso do repositório digital } \\
\text { Huet }\end{array}$ & $\begin{array}{l}\text { Mostrar como um ambiente } \\
\text { informacional digital pode ser } \\
\text { projetado para atender aos } \\
\text { surdos }\end{array}$ & 2019 \\
\hline
\end{tabular}

A partir da análise da tabela 2, percebemos a abrangência interdisciplinar das áreas na qual a Visualidade é tida como elemento mediador da aprendizagem (Figura 2). Verificamos que a visualidade, como sendo uma potencialidade intrínseca dos Surdos, pode ser abordada nos diversos campos de pesquisa. Assim, identificamos dois artigos que se concentram as discussões no campo da Educação, na qual abordam sobre a proposta da educação bilíngue; um artigo no campo da Educação Matemática, na qual apresenta a importância da visualidade no ensino da matemática e um artigo no campo do Ensino de Química, na qual expressa as adaptações visuais na avaliação e os ganhos na aprendizagem.

Figura 2 - Campos de pesquisa em visualidade encontrados nos artigos analisados para a RSL.

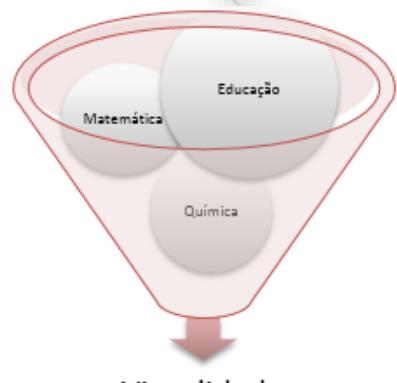

Visualidade

Fonte: O Autor
Quanto aos procedimentos de pesquisa adotados Com fins a compreensão dos procedimentos metodológicos de pesquisa realizados pelos pesquisadores, realizamos uma análise sistemática do subtópico que retrata a metodologia. A seguir, apresentaremos no Quadro 3, o método escolhido e os instrumentos de coleta utilizados.

Quadro 3 - Procedimentos Metodológicos

\begin{tabular}{|c|c|c|c|}
\hline \multicolumn{4}{|c|}{ ARTIGOS } \\
\hline AUTORES & METODO & PARTICIPANTES & INSTRUMENTO \\
\hline $\begin{array}{l}\text { Pivetta, E. M. } \\
\text { Saito, D. S. } \\
\text { Almeida, A. M. P. } \\
\text { Ulbricht, V. R. }\end{array}$ & $\begin{array}{l}\text { Cunho qualitativo. } \\
\text { Pesquisa Exploratória. } \\
\text { Lócus: Escola bilíngue } \\
\text { na cidade do Porto. }\end{array}$ & 6 professores. & $\begin{array}{c}\text { Entrevista } \\
\text { semiestruturada }\end{array}$ \\
\hline Sales, E. R. & $\begin{array}{l}\text { Cunho qualitativo. } \\
\text { Pesquisa exploratória e } \\
\text { descritiva. } \\
\text { Lócus: Escola inclusiva } \\
\text { na cidade de Rio } \\
\text { Claro/SP. }\end{array}$ & $\begin{array}{l}\text { Estudantes do ensino } \\
\text { fundamental }\end{array}$ & $\begin{array}{l}\text { Diário de campo } \\
\text { Filmagens } \\
\text { Entrevistas } \\
\text { Atividades }\end{array}$ \\
\hline $\begin{array}{l}\text { Freitas-Reis, I. } \\
\text { Fernandes, J. M. } \\
\text { Carvalho, V. } \\
\text { Franco-Patrocínio, } \\
\text { S. } \\
\text { Faria, F. L. }\end{array}$ & $\begin{array}{l}\text { Cunho qualitativo. } \\
\text { Pesquisa exploratória. } \\
\text { Lócus: projeto de } \\
\text { extensão da } \\
\text { Universidade Federal de } \\
\text { Juiz de Fora. }\end{array}$ & $\begin{array}{c}\text { Estudantes do ensino } \\
\text { médio }\end{array}$ & $\begin{array}{l}\text { Filmagens } \\
\text { Atividades }\end{array}$ \\
\hline $\begin{array}{l}\text { Paiva, R. O. } \\
\text { Benchimol, A. } \\
\text { Chalhub, T. }\end{array}$ & $\begin{array}{l}\text { Cunho qualitativo. } \\
\text { Pesquisa bibliográfica e } \\
\text { descrtiva| } \\
\text { Lócus: Repositório } \\
\text { digital Huet, }\end{array}$ & - & Repositório Digital \\
\hline
\end{tabular}

Fonte: O Autor

No Quadro 3, podemos perceber as tendências metodológicas escolhidas pelos pesquisadores. Verificamos que todas as pesquisas convergiam a uma abordagem qualitativa, uma vez que o objeto de análise, a visualidade, é melhor compreendida a partir desta escoIha, pois interpreta-se os fenômenos subjetivos.

Além disso, identificamos que os instrumentos de pesquisa utilizados pelos pesquisadores, foram escolhidos a partir da especificidade do estudo e dos sujeitos analisados. Neste caso, para a análise das potencialidades da visualidade, foram necessárias videogravações para que captassem as reações dos estudantes, que serviram como documentação de pesquisa para análise de dados.

\section{Quanto aos resultados de pesquisa}

Como último enfoque da análise sistemática, elencamos os resultados obtidos de cada pesquisa realizada, bem como, as potencialidades da visualidade ex- 
pressas no artigo, em questão.

Quadro 4 - Resultados de pesquisa

\begin{tabular}{|c|c|}
\hline \multicolumn{2}{|r|}{ Artigos } \\
\hline Autores & Resultados \\
\hline $\begin{array}{l}\text { Pivetta, E. M. } \\
\text { Saito, D. S. } \\
\text { Almeida, A. M. P. } \\
\text { Ulbricht, V. R. }\end{array}$ & $\begin{array}{l}\text { Verificou-se que a imagem é uma componente fundamental do } \\
\text { processo de educação bilíngue para alunos surdos, sendo um recurso } \\
\text { fundamental para a aprendizagem. Além disso, o estudo permitiu refletir } \\
\text { sobre um conjunto de preocupações consideradas fundamentais no } \\
\text { desenvolvimento de ambientes acessíveis a surdos, nomeadamente no } \\
\text { que respeita ao design da informação visual e estruturação das } \\
\text { interfaces. }\end{array}$ \\
\hline Sales, E. R. & $\begin{array}{l}\text { Constatou-se que é importante a utilização dos recursos visuais nas } \\
\text { atividades de matemática, por meio de atividades onde os estudantes } \\
\text { surdos possam visualizar, discutir e significar os conceitos dos sinais } \\
\text { específicos da matemática em Libras. }\end{array}$ \\
\hline $\begin{array}{l}\text { Freitas-Reis, I. } \\
\text { Fernandes, J. M. } \\
\text { Carvalho, V. } \\
\text { Franco-Patrocínio, S. } \\
\text { Faria, F. L. }\end{array}$ & $\begin{array}{l}\text { As atividades propostas enfatizaram o potencial de estratégias que } \\
\text { valorizam o aspecto visual para o ensino de surdos. Ademais, permitiu } \\
\text { além da aprendizagem dos conteúdos, a relação dos saberes } \\
\text { estudados com o cotidiano do aluno e com a arte, questões necessárias } \\
\text { para a formação humana. Os resultados mostraram que as atividades } \\
\text { propostas foram significativas para a aprendizagem e contextualização } \\
\text { dos saberes, reforçando o potencial das estratégias como métodos } \\
\text { avaliativos que privilegiam a visualidade dentro do paradigma da } \\
\text { educação de surdos. }\end{array}$ \\
\hline $\begin{array}{l}\text { Paiva, R. O. } \\
\text { Benchimol, A. } \\
\text { Chalhub, T. }\end{array}$ & $\begin{array}{l}\text { Os resultados apontam que, o funcionamento do repositório digital } \\
\text { Huet, vai muito além de somente incluir os surdos por meio de suas } \\
\text { vertentes sensoriais e linguísticas em ambientes digitais, mas } \\
\text { sobretudo, possibilitam autonomia e experiências agradáveis para o } \\
\text { processo de aprendizagem. }\end{array}$ \\
\hline
\end{tabular}

Fonte: O Autor

$\mathrm{Na}$ convergência das constatações encontradas pelos autores, conforme Quadro 4, percebemos que a visualidade propicia o desenvolvimento do pensamento dos estudantes Surdos. E parece ser, um canal de acesso, para formação de conceitos, podendo ser explorado na Educação, tendo em vista, o público alvo, os Surdos.

Sales (2017) ressalta que a característica da visualidade, está para além do uso de instrumentos visuais, mas está interligada com o uso da Libras, para o desenvolvimento do pensamento.

Atrela-se àquilo que Vygotsky (1998, p. 359-360) explicita, o processo de "interpretação da coisa, [...] se dá junto com a percepção [...], a própria percepção de aspectos objetivos isolados deste objeto dependem do sentido, do significado que acompanha a percepção". Ou seja, uma relação dialética de mútua dependência entre percepção e sentido/significado. Simultaneamente com aquilo que vejo, é dada a mim a ordenação categorial da situação visual que constitui, por sua vez, o objeto da percepção.

Percebemos também que, há ganhos para além do processo de aprendizagem, que perpassam a transformação social, autonomia e envolvimento dos estudantes Surdos.

\section{CONSIDERAÇÕES FINAIS}

Compete reforçar, ao final desse artigo, que é de extrema importância se realizar mais pesquisas relacionadas a visualidade como elemento de mediação no processo de aprendizagem, uma vez que nesse estudo foi realizado uma busca na Plataforma Scielo e no Catálogo de Periódicos da CAPES, e foram encontrados somente 4 trabalhos sobre esse tema.

Desse modo, é de suma relevância que os recursos digitais sejam aplicados no ensino, tendo como enfoque estudantes Surdos, seja explorado as potencialidades instrumentais, posto que é por intermédio das experiências visuais que os surdos se apropriam dos conhecimentos. Uma vez que o pensamento visual depende da visão, canal sensorial predominante na atividade dos Surdos. Este canal visual permite à pessoa surda superar limitações de ordem auditiva para construir seu conhecimento de mundo, relacionando língua, instrumento, conceito e significação. Portanto, podemos dizer que o Surdo cria conceitos visuais, tratando a forma como resultado de um ato específico de elaboração e invenção de esquemas de pensamento.

Portanto, contatou-se que as pesquisas que abordam em sua temática Visualidade e aprendizagem de Surdos, são insipientes e que essas precisam ser articuladas mais do que apenas uma ferramenta, mas sim como instrumento pedagógico visual, que perpassam na utilização de artefatos visuais e a língua de sinais.

A partir da RSL, almejamos promover o incentivo do uso desses instrumentos na Educação, uma vez que foram encontrados pouquíssimos estudos relacionados nessa área e explorar as potencialidades do uso na proposição de práticas pedagógicas.

\section{REFERÊNCIAS}

CAMPELLO, A. R. Pedagogia visual na educação dos surdos-mudos. 2008. 169 f. Tese (Doutorado em Educação) - Universidade Federal de Santa Catarina, Santa Catarina, 2008. 
BRASIL. Decreto n. 5.626, de 22 de dezembro de 2005. Regulamenta a Lei $n^{\circ} 10.436$, de 24 de abril de 2002. Disponível em: <http://www.planalto.gov.br/ccivil_03/_ato2004-2006/2005/decreto/d5626.htm>. Acesso em: 23 nov. 2019.

BRASIL. Lei n. 10.436, de 24 de abril de 2002. Dispõe sobre a Língua Brasileira de Sinais (Libras) e dá outras providências. Disponível em: <http://www.planalto.gov. br/ccivil_03/leis/2002//10436.htm>. Acesso em: 05 nov. 2019.

BRASIL. Lei n. 13.005, de 25 de Junho de 2014. Aprova o Plano Nacional de Educação - PNE e dá outras providências. Disponível em: <http://www2.camara.leg.br/ legin/fed/lei/2014/lei-13005-25-junho-2014-778970-publicacaooriginal-144468-pl.html>. Acesso em: 20 nov. 2019.

FELIZARDO, K. et al. Revisão sistemática da literatura em Engenharia de Software: Teoria e prática. Rio de Janeiro: Elsevier, 2017.

FREITAS-REIS, I.; FERNANDES, J. M.; CARVALHO, V.; FRANCO-PATROCÍNIO, S.; FARIA, F. L. Métodos de avaliação para o aluno surdo no contexto do ensino de química. In: X CONGRESO INTERNACIONAL SOBRE INVESTIGACIÓN EN DIDÁCTICA DE LAS CIENCIAS, 10, 2017, Sevilla. Revista de Investigación y experiencias didácticas, 2017. p. 4009-4014.

MARQUES, M. O. A escola no computador: Linguagens rearticuladas, educação outra. ljuí: Unijuí, 1999.

PAIVA, R. O. DE; BENCHIMOL, A.; CHALHUB, T.; FURTADO, C. C. Breves apontamentos sobre um repositório digital biligue (Português-Libras): o caso do repositório digital Huet. Informação \& Sociedade: Estudos, v. 29, n. 1, 27 mar. 2019.

PIVETTA, E. M.; SAITO, D. S.; ALMEIDA, A. M. P.; ULBRICHT, V. R. Contribuições para o design de interface de um Ambiente Virtual de Ensino Aprendizagem acessível a surdos. Brazilian Journal of Information Design, v. 10, n. 2, p. 193, 2013.

REILY, L. As imagens: O lúdico e o absurdo no ensino de arte para pré-escolares surdos. In I. Silva, S. Kauchakje, \& Z. Gesueli (Ed.). Cidadania, surdez e linguagem: Desafios de realidades (p.161-192). São Paulo: Plexus Editora, 2003.

SALES, E. R. A visualização no ensino de matemática: Uma experiência com alunos surdos. Revista Espaço, v. 40 , n. $1,2017$.

VYGOTSKY, L. S. A construção do pensamento e da linguagem. São Paulo: WMF Martins Fontes, 2009. 